\title{
Cambios oculares durante el embarazo
}

\section{Ocular changes during pregnancy}

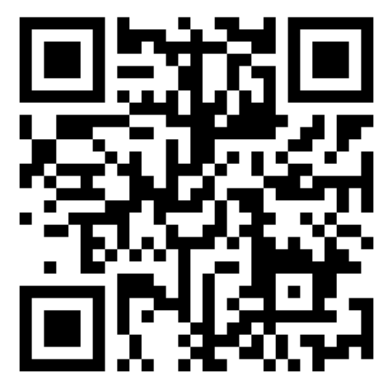

Recibido 17/07/2021
Corregido 10/08/2021

${ }^{1}$ Dra. Andrea Cubillo Espinoza

Hospital San Vicente de Paúl, Heredia, Costa Rica

(iD) https://orcid.org/0000-0002-1214-2095

${ }^{2}$ Dr. Eduardo Chacón Barrantes Hospital San Vicente de Paúl, Heredia, Costa Rica

(iD) https://orcid.org/0000-0002-1180-048X

\section{RESUMEN}

El ojo es uno de los órganos en el cuerpo humano más pequeño y complejo, y como muchos otros órganos del cuerpo, presenta cambios en el embarazo, también puede verse afectado durante este periodo. Sin embargo, en la mayoría de las veces hay cambios fisiológicos, y menos común, patológicos. Es sabido que los cambios oculares durante el embarazo se ven limitados prácticamente al conocimiento de un cirujano oftalmólogo, por lo que es importante mencionar las principales características de estos, y destacar los cambios que pudiesen ser patológicos, de manera que se pueda contar con una guía y conocimiento claro, desde el primer nivel, hasta el médico gineco-obstetra. En este artículo, se revisará la literatura y resumen de los últimos cinco años sobre los cambios oculares durante el embarazo.

PALABRAS CLAVE: cambios oculares; embarazo; patología ocular; preeclampsia; eclampsia; retinopatía.

\section{ABSTRACT}

The eye is one of the most complex and smallest organs, and as many other organs in the human body, it presents changes during pregnancy. Nevertheless, in most cases these changes appear to be physiologic rather than pathologic. It's known that ocular changes during pregnancy are mostly limited to an ophthalmologist knowledge, and because of this it is important to mention the principal characteristics, and highlight the changes that could be pathologic, in a way that general practitioners and ob-gyns can have knowledge and a guideline. In this article we proceed to review the literature and summarize the most important points of ocular changes over the past five years. 
KEYWORDS: ocular changes; pregnancy; ocular pathology; preeclampsia; eclampsia; retinopathy.

${ }^{1}$ Médica general, graduada de la Universidad de Iberoamérica de Costa Rica (UNIBE). Cód. MED12102. Correo: dra.cubillo@gmail.com

${ }^{2}$ Médico cirujano oftalmólogo, graduada de la Universidad de Costa Rica (UCR). Cód. MED9160. Correo: eduardochaconb@hotmail.com

\section{INTRODUCCIÓN}

El embarazo es un estado fisiológico, donde una mujer lleva un feto por aproximadamente nueve meses. Es bien sabido que los cambios son fisiológicos en su mayoría; no obstante, existen también condiciones patológicas que se pueden desarrollar o agravar, en especial por la alteración de los parámetros hormonales y hemodinámicos de la mujer embarazada, que podrían repercutir en la función ocular (1). Debido a lo anterior y sumándole la retención de líquidos, se pueden llegar a producir una serie de alteraciones morfológicas, que se manifestarán en forma de síntomas visuales como alteración de la agudeza o del campo visual. Algunos de estos cambios pueden incluir alteraciones en la circulación ocular, aumento en el grosor coroideo subfoveal en pacientes embarazadas sanas (2). Las estructuras afectadas durante el embarazo incluyen: pestañas, conjuntiva, córnea, cristalino, retina, nervio óptico y órbita (3).

Se ha utilizado recientemente el desarrollo de la angiografía por tomografía de coherencia óptica (OCTA), que permite evaluar la microvasculatura de manera no invasiva, y de la misma manera evaluar tridimensionalmente los vasos sanguíneos de la retina y el disco óptico (2).

Algunas patologías preexistentes en la retina pueden verse afectadas en el embarazo por enfermedades como la diabetes gestacional (DG) y la eclampsia (responsable de la coriorretinopatía serosa central). También se ha observado que los cambios hormonales pueden llevar a cambios en la curvatura de la córnea, la exacerbación del queratocono (asociado a las elevaciones estrogénicas propias de la condición), y la disminución de la presión intraocular, con una mejoría leve en el control del glaucoma (3).

En esta revisión se realizará una revisión de los cambios oculares, tanto patológicos como fisiológicos, durante el embarazo, mediante una revisión sistemática basada en investigaciones en los últimos años.

\section{MÉTODO}

Se realizó el estudio bibliográfico de 28 artículos, de los cuales se utilizaron 15 referencias bibliográficas actualizadas en los últimos cinco años. Se utilizaron palabras clave como: cambios oculares, embarazo, miopía, cambios fisiológicos en el embarazo. La búsqueda fue realizada en distintos medios de información: PubMed, UpToDate, Google Scholar, Medscape. La búsqueda incluyó: artículos sobre miopía, cambios fisiológicos y patológicos en el embarazo, OCT, preeclampsia, eclampsia. Se encontraron documentos entre artículos científicos originales, guías clínicas de práctica, libros y revistas científicos, en idiomas inglés y español.

\section{CAMBIOS FISIOLÓGICOS DURANTE EL EMBARAZO}

\section{Párpados y conjuntiva}

El cloasma, conocido también como "la máscara del embarazo" se presenta en el 
$90 \%$ de los embarazos (2), y es un proceso mediado por hormonas (1), que se caracteriza por un aumento en la pigmentación de las mejillas y zona periocular (párpado inferior) y dorso nasal (4).

La hemorragia subconjuntival (hiposfagma) es un hallazgo inocuo que se observa en aproximadamente un $10 \%$ de las mujeres durante y posterior al parto, y que no requiere ningún abordaje, además de medición de la presión arterial (5); su causa se presume está asociada a un aumento en la granularidad y consecuente viscosidad sanguínea. Existen grandes estudios donde se indica que el melanoma cutáneo no se ve afectado durante el embarazo; sin embargo, reportes más recientes indican lo contrario. El melanoma conjuntival es un tumor raro, con una incidencia de menos de 1/1000000 por año. En la literatura se han descrito únicamente dos casos de melanoma conjuntival, y ambos diagnosticados durante el tercer trimestre de embarazo (6).

\section{Película lagrimal}

Los cambios hormonales son los más prominentes durante el embarazo. Entre ellos se encuentra la elevación de testosterona, sobre todo en el primer trimestre. Hay un aumento concurrente de la globulina transportadora de hormonas, con una reducción circulante activa y libre de testosterona. La secreción de estrógeno y progesterona también se ve incrementada durante este periodo, siendo más prominente en el tercer trimestre del embarazo, que conlleva al ojo seco (7).

Los mecanismos que llevan a presentar ojo seco durante el embarazo incluyen lo siguiente (7):

1. La interacción entre los niveles de estrógeno y testosterona. $\mathrm{La}$ testosterona mejora el desarrollo y la diferenciación en las glándulas de meibomio y el estrógeno. Por otro lado, promueve la muerte acinar, llevando a la reducción en el tamaño y la secreción de la glándula.

2. El embarazo mejora la reactividad inmune de la prolactina, transformándola en factor de crecimiento beta 1 y factor de crecimiento epidermoide en las células ductales, que pueden causar daño directo a las células acinar lagrimales.

3. El aumento en la viscosidad está atribuido al componente de lisozima en la película lagrimal que causa el ojo seco.

4. El ojo seco, durante el embarazo, también es atribuido a la deshidratación en el primer trimestre causada por los vómitos y náuseas. Los lentes de contacto deben evitarse durante el embarazo, para evitar mayor resequedad en el ojo.

También, durante el embarazo se produce una disrupción de las células acinares y una redistribución de linfocitos en sitios interacinares, produciendo un ojo seco secundario al embarazo (4). La paciente se quejará de sensación de cuerpo extraño, irritación, prurito, ardor y enrojecimiento leve (inyección conjuntival difusa leve).

\section{Córnea y cambios refractarios}

La córnea actúa como barrera de protección, donde se aumenta su grosor, y se ha descrito ampliamente la sensibilidad disminuida de la córnea, especialmente durante el tercer trimestre y su remisión en el periodo postparto (3). Estos cambios corneales causan cambios refractivos temporales, y a los cuales debe ponérseles especial atención sobre la decisión de 
realizar cirugía refractiva. Es importante que, de considerarse cirugía, deberá posponerse hasta asegurar una estabilidad refractiva.

La morfología de la córnea y de la cámara anterior durante el tercer trimestre cambian en relación con el periodo postparto (8). El edema corneal durante el embarazo es uno de los fenómenos mejor descritos sobre este periodo, siendo provocado por un aumento en el contenido acuoso, que puede causar intolerancia a los lentes de contacto.

Por otro lado, se ha mencionado, en algunos estudios, que el queratocono ha progresado durante el embarazo, y que el mismo es persistente incluso posterior al parto $(3,9)$. En el estudio realizado por el Dr. Naderan, en el cual se dio un seguimiento un grupo de 22 ojos con un queratocono estable de dos años de evolución, y se evaluaron en el periodo de preembarazo (con el plan de quedar embarazadas), al tercer trimestre (34 semanas) y seis meses postparto, se concluyó que hubo un aumento en el progreso de la enfermedad (evidenciada en aumento de la curvatura y adelgazamiento de la córnea) en todas las etapas de la evaluación, e inclusive se sugiere que se deberían considerar medidas preembarazo (fortalecimiento del colágeno corneal por medio de la riboflavina, procedimiento llamado cross-linking) (10).

De la miopía, siendo uno de los problemas refractivos más comunes, aún no se ha identificado que el embarazo sea un factor de riesgo para su progresión, pero se cree que los errores refractivos se ven temporalmente elevados (11). Cabe mencionar que la miopía alta (mayor de 6.00 dioptrías) no es una contraindicación para el parto vaginal, ya que no se he evidenciado un aumento en el riesgo de desprendimientos de retina (12), e inclusive en la actualidad no existe una contraindicación establecida para no seleccionar la vía por parto vaginal ante factores de riesgo de desprendimientos de retina.

\section{Retina}

El crecimiento fisiológico de la hipófisis puede resultar en cambios en el campo visual, que varían desde hemianopsia bitemporal, reducción del campo visual concéntrico hasta hemianopsia homónima (7). Sin embargo, para que exista un cambio en el campo visual causado por este crecimiento, debe existir una relación anormal entre el quiasma óptico y la hipófisis (4). Estos cambios se han documento que se revierten aproximadamente a los diez días postparto (8).

La coroides es un tejido altamente vascularizado, el cual es susceptible a los cambios hormonales y hemodinámicos en el embarazo (13). El grosor de la coroides se ha reportado que se puede ver aumentado en este periodo, con un aumento máximo durante el segundo trimestre de embarazo (7).

\section{Cristalino}

Por otro lado, el cristalino está constituido, en un $65 \%$ de su volumen, por agua (14); por lo tanto, el aumento de volumen puede resultar en el desarrollo o exacerbación de cataratas durante el embarazo (3).

\section{Presión intraocular}

Según la The American Academy of Ophthalmology, la presión intraocular está definida como la presión generada por la circulación del humor acuoso que se encuentra dentro del ojo.

Por lo general, la presión normal del ojo se considera entre los 10 y los $20 \mathrm{mmHg}$ (15). El término presión intraocular normal incluye 
aquel nivel que usualmente no lleva a un daño glaucomatoso y daño a la visión. Estudios iniciales referían que los niveles fluctuantes de estrógeno, progesterona, relaxina y HCG eran causa del descenso en la presión intraocular (7).

Existe una disminución progresiva de la presión intraocular conforme avanza el embarazo de hasta del $19 \%$, siendo mayor entre las semanas 12 y 18 (4). Los mecanismos por los cuales esto sucede no han sido definidos; no obstante, se presume que se produce un aumento en el drenaje de humor acuoso junto a una disminución de la resistencia vascular sistémica. Se dice que la presión intraocular se mantiene en un rango por debajo de 2 a $3 \mathrm{mmHg}$ durante el embarazo por el efecto de la progesterona (4), así como la relaxina, que produce disminución del drenaje acuoso y disminución de la presión venosa epiescleral (16).

Si bien es cierto el mecanismo exacto por el que la presión intraocular se ve disminuida durante el embarazo, varios factores han sido propuestos, como la disminución de humor acuoso, disminución de la rigidez escleral como resultado del aumento de la elasticidad del tejido, menor presión venosa epiescleral, debido al descenso de la resistencia vascular y acidosis general durante el embarazo (3).

\section{Cambios patológicos durante el embarazo}

\section{Cambios neurooftalmológicos en el embarazo (7)}

- Trombosis de senos venosos.

- Adenoma y meningiona pituitario.

- Neuritis óptica y neuropatía.

- Pseudotumor cerebri.

\section{Complicaciones por trastornos hipertensivos en el embarazo, preeclampsia y eclampsia}

La hipertensión gestacional (HG) se define como la elevación de la presión arterial por arriba de $140 \mathrm{mmHg}$ pero menor de 160 $\mathrm{mmHg}$, o PA diastólica mayor de $90 \mathrm{mmHg}$ pero menor de $110 \mathrm{mmHg}$, tomadas en dos ocasiones en un intervalo de al menos cuatro horas, pero no mayor a siete días de diferencia, y que se presenta posterior a las 20 semanas de gestación (17). Entre un $40 \%-100 \%$ de las pacientes demuestran retinopatía variable, pero menos de la mitad son sintomáticas (4). La preeclampsia se define como la hipertensión gestacional más proteinuria $>300 \mathrm{mg}$ en orina de 24 horas. La eclampsia es la aparición de convulsiones o coma, sin relación con otras afecciones cerebrales $(7,17)$. Esta condición puede llevar a hiperperfusión, y como consecuencia hiperemia en el sistema ocular, desarrollando retinopatía hipertensa y neuropatía óptica (3).

Algunos hallazgos de la retinopatía pueden incluir hemorragias, papiledema y acumulación de fluido subretiniano (7); además, complicaciones como hemovítreo, ceguera cortical, desprendimiento seroso de la retina y oclusión venosa central $(3,7)$.

Las pacientes que presentan estos trastornos hipertensivos presentan mayor riesgo de desarrollar disfunción coroidea y retiniana, que puede llevar incluso a pérdida de la visión (7). Los síntomas pueden incluir desde disminución de la agudeza visual, diplopía, escotomas hasta fotopsias (5).

La ceguera cortical puede ocurrir en el tercer trimestre o posterior al parto, en los casos de preeclampsia con criterios de severidad o eclampsia, debido al compromiso del lóbulo occipital. El grado de retinopatía dependerá directamente del grado de severidad del 
trastorno hipertensivo, y es por esto la importancia de que tanto el médico ginecoobstetra como el oftalmólogo se vean involucrados en su manejo $(3,5,7)$.

\section{Coriorretinopatía serosa central}

La coriorretinopatía serosa central (CSC) está definida como un desprendimiento espontáneo y localizado de la retina neurosensorial de la mácula, la cual causa síntomas de visión borrosa, escotomas centrales, metamorfopsias y micropsias (4). Es causada por un aumento de catecolaminas en el embarazo, que causa un incremento en los niveles de cortisol en el tercer trimestre (7). Adicionalmente, el estrés vasomotor del embarazo puede llevar a la disfunción coroidal, que juega el rol primario en la fisiopatología de esta entidad (7).

Los estudios han descrito la coriorretinopatía serosa central como una complicación ocular con una incidencia de $0.008 \%$ (7). Es conocida por afectar mayormente a los hombres, por lo que su diagnóstico en el embarazo puede pasarse por alto (5). Generalmente, se resuelve espontáneamente posterior al parto, con mínima o ninguna secuela. Previamente, Quillen y Kitzmann et al. habían reportado un $9 \%$ de incidencia en pacientes embarazadas, e informaron que un 5\% habían desarrollado desprendimiento de retina y pérdida de la visión severa (7). La CSR por lo general resuelve posterior al parto, pero con mayor riesgo de recurrencia en futuros embarazos $(4,5)$.

\section{Oclusiones retinianas}

Retinopatía de Purtscher-like esta etiología está relacionada con la agregación granulocítica inducida por el complemento y la oclusión vascular (4), y puede ser arterial o venosa. La retinopatía arterial se presenta en el postparto inmediato (3). La incidencia es baja, en comparación con una población de mujeres de la misma edad, con solo 33 casos en 1.8 millones de partos (7).

En la oftalmoscopia generalmente se observan múltiples parches retinianos 0 exudados algodonosos confluentes alrededor del disco óptico, ambos con hemorragias retinianas superficiales (5). Hallazgos menos comunes como ramas y oclusiones de la retina centrales, así como la oclusión venosa, han sido reportados durante el embarazo, probablemente secundarios al estado de hipercoagubilidad o a un émbolo del líquido amniótico (3), así como por la activación de complemento y agregación leucocitaria, que pueden formar émbolos retinianos (7).

La coagulación intravascular diseminada (CID) es un síndrome adquirido, así como una complicación del síndrome de HELLP. LA CID tiene causas variables en el embarazo, como lo son la preclamsia, la eclampsia, el embolismo de líquido amniótico, la muerte intrauterina, la infección intrauterina, el aborto séptico, entre otros. La estructura ocular mayormente afectada en la CID es la coroides. La oclusión por un trombo de los coriocapilares está asociada a la CID en el embarazo (3), que puede llevar a la disrupción del epitelio pigmentario de la retina, causando desprendimiento seroso de la retina $(3,4)$. El embarazo es conocido por ser un estado de hipercoagubilidad para la mujer, por lo que puede llevar a desarrollar una púrpura trombocitopenia y un síndrome antifosfolípido. Estos están asociados también a la oclusión venosa o arterial de la retina (7).

\section{Problemas oftalmológicos relacionados con la labor de parto}

La maculopatía, por la valsalva realizada durante el parto, causa un aumento de la 
presión venosa, que puede llevar a la paciente a referir visión borrosa, provocada por una hemorragia vítrea, subretiniana, o retiniana. Si existe desprendimiento de retina, se recomienda un parto por fórceps o cesárea (7). Sin embargo, en un estudio realizado con 69 mujeres en Polonia, que presentaban distintos problemas de retina, se concluyó que hay que individualizar cada caso previo a definir vía de parto (12).

El síndrome de Sheehan aparece como el resultado de isquemia necrótica de la hipófisis debido a hemorragia severa en el postparto (7). Puede estar acompañado de cefalea súbita, pérdida del campo visual y de la visión, y/o oftalmoplejía. Se presentan típicamente sobre el cuadrante superior bitemporal. Existe compresión del seno cavernoso que afecta el tercer, cuarto y sexto par craneal lo, cual causa ptosis, diplopía, midriasis y desviación lateroinferior del globo ocular; este incluye las fibras simpáticas que causan el síndrome de Horner (3).

\section{CONCLUSIONES}

Dentro de la inmensa gama de cambios y adaptaciones en el embarazo, los cambios oculares no son una excepción, y se presentan en prácticamente todas las estructuras del ojo y de diferente manera, según la etapa de la gestante. La mayoría de los cambios obedece a procesos fisiológicos, que en algunas ocasiones pueden causar ciertas molestias en las pacientes, en donde médicos tratantes deben brindar la atención y saber cuándo algo amerite una atención especializada.

Un ejemplo de dichos cambios se encuentra en el cloasma, como una pigmentación facial y de anexos oculares, la cual suele ser autolimitada, al igual que el edema corneal y ojo seco. Los cambios en el poder refractivo del ojo son de manejo conservador, y no se debe recomendar cambio de graduaciones ni cirugías de corrección refractiva durante este periodo. De diferente abordaje serían las lesiones oculares causadas por la preclamsia, que podría producir cuadros de hemorragias en retina y edema de disco óptico. Otra afectación del segmento posterior ocular sería la CSC, la cual se manifiesta como alteraciones en la forma y tamaño de los objetos, causada por un proceso exudativo en la mácula y la consecuente disrupción de la capa de fotorreceptores.

Es importante que el médico tratante esté al tanto de los cambios fisiológicos, y también de aquellos que puedan verse alterados durante el embarazo, de manera que se pueda realizar un diagnóstico correcto, y tratarse tempranamente. Estratégicamente, debe ponerse especial atención a aquellas pacientes que aquejan diferentes síntomas oculares, sobre todo pérdida súbita de la visión. De esta manera, se logrará tanto el conocimiento de los cambios oculares normales, así como los signos de alarma a los cuales se deben poner mayor atención y estudiar con mayor precisión.

\section{Los autores declaran no tener conflicto de interés.}

\section{REFERENCIAS}

1. Agrawal N, Agarwal LT, Lavaju P, Chaudhary SK. Physiological Ocular Changes in Various Trimesters of Pregnancy. Nepal J Ophthalmol. 20 de noviembre de 2018;10(1):16-22.

2. Kızıltunç $P B$, Varlı $B$, Büyüktepe TÇ, Atilla $H$. Ocular vascular changes during pregnancy: an optical coherence tomography angiography study. Graefes Arch Clin Exp Ophthalmol. febrero de 2020;258(2):395-401.

3. Naderan M. Ocular changes during pregnancy. J Curr Ophthalmol. septiembre de 2018;30(3):20210.

4. Marcos-Figueiredo $P$, Marcos-Figueiredo A, Menéres $\mathrm{P}$, Braga J. Ocular Changes During 
Pregnancy. Rev Bras Ginecol E Obstetrícia RBGO Gynecol Obstet. enero de 2018;40(01):032-42.

5. Kalogeropoulos D, Sung VC, Paschopoulos M, Moschos MM, Panidis P, Kalogeropoulos C. The physiologic and pathologic effects of pregnancy on the human visual system. J Obstet Gynaecol. 17 de noviembre de 2019;39(8):1037-48.

6. Al-Jamal RT, Mudhar HS, Currie Z, Rennie IG, Salvi SM. Conjunctival Melanoma during Pregnancy. Ocul Oncol Pathol. 2017;3(2):101-5.

7. Morya A, Gogia S, Gupta A, Prakash S, Solanki K, Naidu A. Motherhood: What every ophthalmologist needs to know. Indian J Ophthalmol. 2020;68(8):1526.

8. karol Taradaj, Tomasz Ginda, Piotr MAciejewics, Piotr Ciechanowicz, Barbara Suchonska, Marta Hajbos, et al. Pregnancy and the eye. Changes in morphology of the cornea and the anterior chamber of the eye in pregnant woman. Medica. 2018;89(no.12):695-9.

9. Yang Y, Ye J, Ng TK, Wu N, Sun X, Chen Y, et al. Changes in corneal biomechanics during pregnancy in Chinese healthy female. Eur $\mathrm{J}$ Ophthalmol. marzo de 2021;31(2):361-6.

10. Maier $\mathrm{P}$, Reinhard $\mathrm{T}$, Kohlhaas $\mathrm{M}$. Corneal Collagen Cross-Linking in the Stabilization of Keratoconus. Dtsch Aerzteblatt Online [Internet]. 15 de marzo de 2019 [citado 16 de julio de 2021]; Disponible en: https://www.aerzteblatt.de/10.3238/arztebl.2019. $\underline{0184}$

11. Fernández-Montero A, Bes-Rastrollo M, MorenoMontañés J, Moreno-Galarraga L, MartínezGonzález MÁ. Effect of pregnancy in myopia progression: the SUN cohort. Eye. julio de 2017;31(7):1085-92.

12. Joanna Moneta- Wielgos, Michal Lipa, Joanna Brydak-Godowska, MArek Rekas, Miroslaw Wielgos. Opthalmological and obstetric management in pregnant women with retinal disorders. Ginekol Pol. 2019;90:285-8.

13. Wu F, Schallhorn JM, Lowry EA. Refractive status during pregnancy in the United States: results from NHANES 2005-2008. Graefes Arch Clin Exp Ophthalmol. marzo de 2020;258(3):663-7.

14. Miesfeld JB, Brown NL. Eye organogenesis: A hierarchical view of ocular development. En: Current Topics in Developmental Biology [Internet]. Elsevier; 2019 [citado 15 de julio de 2021]. p. 351-93. Disponible en: https://linkinghub.elsevier.com/retrieve/pii/S0070 21531830108X

15. Salmon JF, Kanski JJ. Kanski's clinical ophthalmology: a systematic approach. 2020.
16. Akkara J, Kuriakose A. Commentary: Pregnancy hormone to control intraocular pressure? Indian J Ophthalmol. 2020;68(10):2121.

17. Gabbe SG, Niebyl JR, Simpson JL. Obstetricia: embarazos normales y de riesgo. Madrid: Elsevier; 2019. 